\title{
Perfil ePidemiológico dos usuários da Rede de Saúde Mental do Município de IGUATU, CE
}

\section{Marcos Danúbio Alves de Carvalho ${ }^{1}$; Hélder Oliveira e Silva ${ }^{2}$; Leila Vieira Rodrigues ${ }^{3}$}

Este artigo apresenta o perfil epidemiológico dos usuários da rede de saúde mental do município de Iguatu, CE. Trata-se de estudo descritivo com abordagem quantitativa. Foram avaliados os prontuários de 143 usuários, cadastrados e atendidos nos três Centros de Atenção Psicossocial (CAPS) do município. Constatou-se que a maioria dos usuários chega aos serviços por iniciativa da família, possuem baixa escolaridade e renda média variando entre 1 e 2 salários mínimos. Conclui-se que os serviços avaliados necessitam fortalecer a prática da clínica ampliada, intensificando as ações coletivas, reduzindo a medicalização e efetivando a integração dos usuários com suas redes de apoio.

Descritores: Saúde Mental; Epidemiologia; Serviços de Saúde.

\section{Epidemiological Profile of Users of the Municipal Mental Health Care NETWORK OF IGUATU, CEARÁ, BRAZIL}

This article presents the epidemiological profile of users of the municipal mental health care network of Iguatu, Ceará state, Brazil. This is a descriptive study using a quantitative approach. Evaluations were performed on the medical records of 143 users enrolled and attended at the three Psychosocial Care Centers (CAPS) in Iguatu. It was found that most users seek the service encouraged by their families, they have low education levels and an average income ranging between 1 and 2 minimum salaries. It is concluded that the evaluated services need to strengthen the practice of the extended clinic, making collective actions more intensive, reducing medicalization and effectively integrating users with their support networks.

Descriptors: Mental Health; Epidemiology; Health Services.

\section{Perfil epidemiológico de los usuarios de la Red de Salud Mental del Municipio DE IGUATU, CE, BRAZIL}

Este artículo presenta el perfil epidemiológico de los usuarios de la red de salud mental del municipio de Iguatu, CE. Se trata de un estudio descriptivo con abordaje cuantitativo. Fueron evaluadas las historias clínicas de 143 usuarios registrados y atendidos en los tres Centros de Atención Psicosocial (CAPS) del municipio. Se constató que la mayoría de los usuarios llegan a los servicios por iniciativa de la familia, poseen baja escolaridad y renta media, variando entre 1 y 2 salarios mínimos. Se concluye en que los servicios evaluados necesitan fortalecer la práctica de la clínica ampliada, intensificando las acciones colectivas, reduciendo la medicalización y haciendo efectiva la integración de los usuarios con sus redes de apoyo.

Descriptores: Salud Mental; Epidemiología; Servicios de Salud.

1-Enfermeiro, Universidade Regional do Cariri.E-mail: rammsteinm@ hotmail.com

2-Enfermeiro, Mestre em Saúde Coletiva, Professor Colaborador do Curso de Enfermagem da Universidade Regional do Cariri.E-mail: heldersilva@terra.com.br

3-Enfermeira, Especialista em Saúde da Família pela Universidade Estadual do Ceará. E-mail: leilavrodrigues@yahoo.com.br

Endereço para correspondência: Universidade Regional do Cariri, Rua Dom Quintino, S/N, Bariro São Sebastião, 63500000, Iguatu, CE, Brasil.

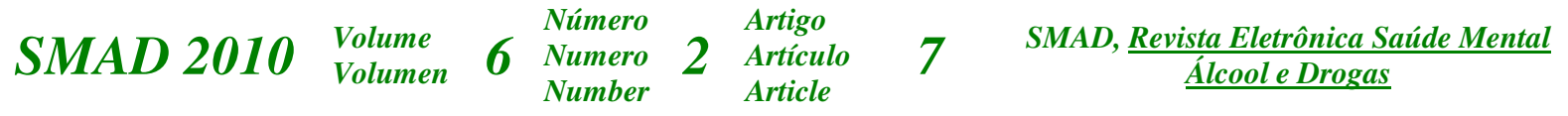




\section{Introdução}

Os Centros de Atenção Psicossocial (CAPS) caracterizam-se por serviços de saúde mental regionalizados, que prestam cuidados intermediários entre o ambulatório e a internação, atendendo a clientela adstrita à sua localização, integrado a uma rede de cuidados, visando garantir a referência e contrarreferência de casos de distintas complexidades ${ }^{(1-2)}$.

Os CAPS devem prestar atendimento individual, medicamentoso, psicoterápico, grupal, oficinas terapêuticas, atividades comunitárias e socioterápicas, visitas domiciliares e atendimento à família, enfocando a integração do paciente ao seu meio social ${ }^{(1)}$.

Nos últimos anos, o número de CAPS vem crescendo em diversas cidades brasileiras, destinando-se a acolher os pacientes com transtornos mentais, estimular sua integração social e familiar, apoiá-los em suas iniciativas de busca da autonomia e oferecer-lhes atendimento sob perspectiva integral. Sua característica principal é buscar integrá-los a um ambiente social e cultural concreto, designado como seu "território", o espaço da cidade onde se desenvolve a vida quotidiana de usuários e familiares ${ }^{(3)}$.

Apesar da ampliação da rede de saúde mental em todo país e de maior oferta de serviços, ainda não é possível contemplar toda a demanda que, muitas vezes, não consegue acompanhamento especializado e tem dificuldade de acesso aos CAPS e aos demais serviços ofertados no sistema de saúde ${ }^{(4)}$.

A Organização Mundial de Saúde (OMS) preconiza que, para melhorar a saúde mental, deve-se investir na produção de dados concretos sobre os serviços e recursos existentes e definir estratégia de avaliação contínua de toda nova atividade. Para se alcançar o aperfeiçoamento dos serviços de saúde mental, se faz necessária uma "atitude 
epidemiológica", que possibilite ratificar que o manejo do sofrimento psíquico não se limita à aplicação meticulosa de técnicas, mas necessita, também, de avanços na acessibilidade, na humanização e na criação de estratégias de promoção à saúde, que abordem de forma integral as questões sociais e de relacionamento interpessoal, envolvendo os usuários desses serviços $^{(5)}$.

Diante da magnitude dos transtornos mentais, torna-se relevante incentivar investigações epidemiológicas na perspectiva de subsidiar informações concretas da área de saúde mental, bem como despertar em outros profissionais o interesse pela produção dos dados que caracterizem melhor a população que está sendo trabalhada. Nesse contexto, o presente estudo descreve o perfil epidemiológico dos usuários da rede de saúde mental, assistidos nos CAPS do município de Iguatu, CE.

\section{Material e Métodos}

Trata-se de estudo descritivo com abordagem quantitativa. A pesquisa foi realizada no município de Iguatu, CE, localizado na região centro-sul do Estado do Ceará, a 380km da capital Fortaleza, e possui população estimada em 92.260 habitantes $^{(6)}$.

O município de Iguatu conta com três CAPS, que possuem contingente de, aproximadamente, 5.000 pacientes cadastrados. Foram incluídos no estudo usuários com prontuários devidamente preenchidos e que haviam sido acompanhados em um dos serviços da rede de saúde mental, no período de julho a dezembro de 2008. Foi utilizada amostra de 
conveniência, que contou com 58 prontuários do CAPS Geral, 47 do CAPS AD (álcool e drogas) e 38 do CAPSi (infantil), totalizando 143 registros avaliados.

As variáveis investigadas foram: sexo, idade, procedência, escolaridade, estado civil, ocupação, renda, origem de encaminhamento ao CAPS, data do início do acompanhamento, diagnóstico, frequência de acompanhamento, uso de medicação contínua, história de internação psiquiátrica e participação em atividades terapêuticas.

A coleta de dados ocorreu no período de fevereiro a março de 2009. Os dados foram analisados no programa Statistical Package for the Social Sciences (SPSS), versão 11.0. A pesquisa foi submetida ao Comitê de Ética da Faculdade de Medicina de Juazeiro (FMJ), tendo sido aprovada sob Parecer 20090002FR238823, respeitando os termos da Resolução 196/96, do Conselho Nacional de Saúde (CNS).

\section{Resultados}

No CAPS Geral, a faixa etária dos pacientes avaliados variou de 18 a 72 anos, com média de idade de 42 anos. No CAPS AD, essa faixa etária variou de 19 a 74 anos, com média de idade de 41,9 anos. No CAPSi, a faixa etária variou de 3 a 17 anos. A idade média foi de 10,8 anos.

A Tabela 1 apresenta número e percentual de dados sociodemográficos dos usuários atendidos nos CAPS AD, CAPSi e CAPS Geral. Foram consideradas as variáveis: sexo, escolaridade, renda familiar e estado civil.

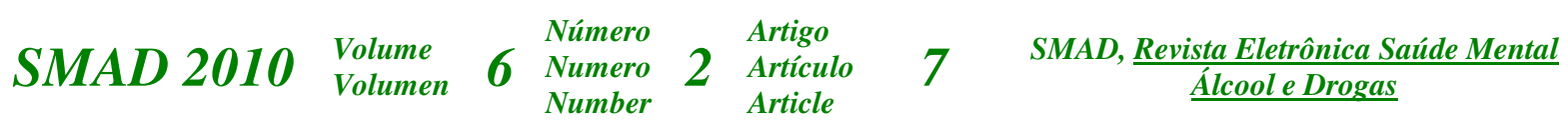


Tabela 1 - Dados sociodemográficos dos pacientes atendidos nos CAPS de Iguatu, CE, 2009

\begin{tabular}{|c|c|c|c|c|c|c|}
\hline \multirow[t]{2}{*}{$\begin{array}{c}\text { Dados } \\
\text { sociodemográficos }\end{array}$} & \multicolumn{2}{|c|}{ CAPS AD } & \multicolumn{2}{|c|}{ CAPSi } & \multicolumn{2}{|c|}{ CAPS Geral } \\
\hline & $\mathbf{n}$ & $\%$ & $\mathbf{n}$ & $\%$ & $\mathbf{n}$ & $\%$ \\
\hline \multicolumn{7}{|l|}{ Sexo } \\
\hline Masculino & 40 & 85,1 & 27 & 71,1 & 12 & 20,7 \\
\hline Feminino & 7 & 14,9 & 11 & 28,9 & 46 & 79,3 \\
\hline \multicolumn{7}{|l|}{ Escolaridade } \\
\hline Nenhuma & 13 & 27,6 & 8 & 21,1 & 14 & 24,1 \\
\hline 1 a 3 anos & 3 & 6,4 & 4 & 10,5 & 11 & 18,9 \\
\hline 4 a 7 anos & 20 & 42,6 & 14 & 36,7 & 23 & 39,7 \\
\hline 8 a 11 anos & 8 & 17 & 8 & 21,1 & 4 & 6,9 \\
\hline 12 anos ou mais & 3 & 6,4 & 2 & 5,3 & 6 & 10,4 \\
\hline Não se aplica & - & - & 2 & 5,3 & - & - \\
\hline \multicolumn{7}{|l|}{ Renda familiar } \\
\hline$<1 \mathrm{SM}$ & 17 & 36,1 & - & - & - & - \\
\hline 1 a 2 SM & 24 & 51,1 & - & - & 12 & 20,7 \\
\hline 3 a 4 SM & 1 & 2,1 & - & - & - & - \\
\hline $5 \mathrm{SM}$ ou mais & 2 & 4,3 & - & - & - & - \\
\hline Não especificado & 3 & 6,4 & 38 & 100 & 46 & 79,3 \\
\hline \multicolumn{7}{|l|}{ Estado civil } \\
\hline Solteiro & 28 & 59,6 & 38 & 100 & 22 & 38 \\
\hline Casado & 15 & 31,9 & - & - & 30 & 51,7 \\
\hline União estável & 0 & 0 & - & - & 1 & 1,7 \\
\hline Viúvo & 1 & 2,1 & - & - & - & - \\
\hline
\end{tabular}

SM - salário mínimo

A Tabela 2 traz as frequências absoluta e percentual relacionadas à origem de encaminhamento ao CAPS, frequência de acompanhamento, uso de medicação controlada e história de internação psiquiátrica.

Tabela 2 - Frequência absoluta e percentual em relação à origem de encaminhamento ao CAPS, frequência de acompanhamento, uso de medicação controlada e história de internação psiquiátrica. Iguatu, CE, 2009

SMAD $2010 \begin{aligned} & \begin{array}{l}\text { Volume } \\ \text { Volumen }\end{array} \\ & \text { SMumero }\end{aligned} 2 \begin{aligned} & \begin{array}{l}\text { Número } \\ \text { Nurtigo } \\ \text { Number }\end{array} \\ & \text { Article }\end{aligned} \quad 7 \quad$ SMAD, $\frac{\text { Revista Eletrônica Saúde Mental }}{\underline{\text { Alcool e Drogas }}}$ 


\begin{tabular}{ccccccc}
\hline Dados clínicos & CAPS AD & CAPSi & \multicolumn{2}{c}{ CAPS Geral } \\
\hline & n & $\%$ & n & $\%$ & n & $\%$
\end{tabular}

Origem do encaminhamento ao CAPS

Família

PSF

Hospital geral

Hospital psiquiátrico

Demanda livre

Outros

\section{Acompanhamento profissional}

Diário

Semanal

Quinzenal

Mensal

Bimestral

Trimestral

$3 x$ por semana

\section{Medicação contínua}

$\begin{array}{ccccccc}\text { Sim } & 42 & 89,4 & 18 & 47,4 & 58 & 100 \\ \text { Não } & 5 & 10,6 & 20 & 52,6 & - & -\end{array}$

\section{Internação psiquiátrica}

Sim

Não

Não informado

$\begin{array}{cccccc}22 & 46,9 & 23 & 60,5 & 40 & 69 \\ 5 & 10,6 & 8 & 21,1 & 4 & 6,9 \\ 5 & 10,6 & 3 & 7,9 & 4 & 6,9 \\ - & - & - & - & 4 & 6,9 \\ 10 & 21,3 & - & - & 4 & 6,9 \\ 5 & 10,6 & 4 & 10,5 & 2 & 3,4\end{array}$

$\begin{array}{lllllll}17 & 36,2 & 11 & 28,9 & - & -\end{array}$

$\begin{array}{llllll}3 & 6,4 & 24 & 63,2 & 4 & 6,9\end{array}$

$\begin{array}{llllll}2 & 4,3 & - & - & 2 & 3,4\end{array}$

$25 \quad 53,1 \quad-\quad-\quad 22 \quad 37,9$

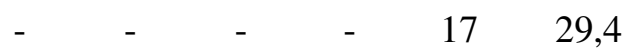

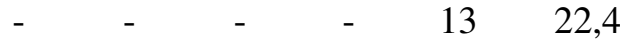

$\begin{array}{lllll}- & - & 3 & 7,9 & -\end{array}$

$\begin{array}{cccccc}1 & 2,1 & - & - & 8 & 13,8 \\ 46 & 97,9 & 38 & 100 & 37 & 63,8 \\ - & - & - & - & 13 & 22,4\end{array}$

Quanto às modalidades terapêuticas empregadas, foi possível observar que 52,4\% $(n=75)$ dos pacientes atendidos nos três CAPS de Iguatu, CE, participaram de atividades terapêuticas individuais e grupais, 46,2\% (n=66) dos prontuários apontaram registros somente de atendimento individual e apenas $1,4 \%(n=2)$ com participação em atividades coletivas.

Observaram-se diferenças nas modalidades terapêuticas empregadas em cada uma das instituições avaliadas. No CAPS AD, 95,7\% $(n=45)$ participaram de atividades terapêuticas SMAD $2010 \begin{aligned} & \text { Volume } \\ & \text { Volumen }\end{aligned} \quad 6 \begin{aligned} & \begin{array}{l}\text { Número } \\ \text { Numero } \\ \text { Number }\end{array} \\ & 2\end{aligned} \begin{aligned} & \begin{array}{l}\text { Artigo } \\ \text { Artículo } \\ \text { Article }\end{array}\end{aligned} \quad$ SMAD, $\frac{\text { Revista Eletrônica Saúde Mental }}{\underline{\text { Alcool e Drogas }}}$ 
individuais e coletivas. No CAPSi, 44,7\% $(n=17)$ das crianças e adolescentes foram acompanhadas exclusivamente em atendimentos individuais e 50\% $(\mathrm{n}=19)$ receberam atendimento individual e em grupo. No CAPS Geral, $81 \%(n=47)$ dos usuários receberam apenas atendimento individual, enquanto que $19 \%(\mathrm{n}=11)$ eram atendidos individualmente e através de ações coletivas.

Considerando os diagnósticos encontrados nos prontuários dos usuários avaliados, verificou-se que, no CAPS Geral, os transtornos de ansiedade $(n=17)$, esquizofrenia $(n=16)$ e transtorno bipolar $(\mathrm{n}=08)$ foram os diagnósticos mais frequentes. No CAPS AD, os transtornos comportamentais por abuso de álcool $(n=43)$, os transtornos comportamentais por abuso de fumo $(n=19)$, os transtornos mentais por abuso de múltiplas drogas $(n=4)$ e as psicoses não orgânicas não especificadas $(n=4)$ foram os diagnósticos mais descritos. No CAPSi, os diagnósticos mais frequentes foram, respectivamente, os transtornos ansiosos $(n=8)$, os retardos mentais não especificados $(n=7)$, as reações ao stress grave $(n=5)$ e os transtornos de comportamento $(n=4)$.

\section{Discussão}

Neste estudo, os resultados apontam disparidade entre os usuários do CAPS AD e CAPS Geral, quanto ao estado civil. No primeiro serviço, a maior parte dos usuários era solteira, já no segundo predominavam os casados. Em investigação realizada em Portugal, com pacientes com transtornos mentais acompanhados em serviços ambulatoriais, verificou- 
se, quanto ao estado civil, que $63 \%$ dos usuários eram solteiros, $31 \%$ eram casados, $4,4 \%$ divorciadas e $1,6 \%$ eram viúvos ${ }^{(7)}$.

Observou-se que a família foi a principal responsável por encaminhar os usuários aos três Centros de Atenção Psicossocial do município de Iguatu. O trabalho do CAPS só se concretiza através da parceria e da participação familiar, tornando-se fundamental a inserção da família no enfrentamento do sofrimento psíquico, integrando, acolhendo, cuidando e incluindo os atores dessa relação nos espaços cotidianos da vida ${ }^{(8)}$.

Quanto à frequência de acompanhamento profissional, verificou-se que os três serviços seguem as normas recomendadas pelo Ministério da Saúde. Segundo o Manual de Saúde Mental, a frequência de acompanhamento dos usuários nos CAPS dependerá de seu projeto terapêutico, sendo necessário haver flexibilidade, podendo variar de cinco vezes por semana com oito horas por dia a, pelo menos, uma consulta a cada três meses ${ }^{(9)}$.

O que também determina a frequência dos usuários no serviço é o acesso que os usuários têm ao CAPS, o apoio e/ou o acompanhamento familiar e a possibilidade de envolvimento nas atividades comunitárias, organizativas, de geração de renda e trabalho. Os CAPS gerais por ter funcionamento durante 24 horas, em alguns casos, podem oferecer acolhimento noturno ${ }^{(9)}$.

Quanto ao uso de medicação, observou-se que elevado percentual de usuários fazia uso de medicação continuamente. Esses resultados estão próximos aos encontrados em Fortaleza, CE, onde $91 \%$ dos usuários dos CAPS faziam uso rotineiro da terapia medicamentosa ${ }^{(10)}$. Verifica-se que, apesar de os CAPS trazerem nova proposta de atenção ao paciente psiquiátrico, a ênfase no tratamento continua baseada no modelo clínico, centrado na 
doença e "medicalizador", tendo no psicofármaco o principal instrumento de intervenção terapêutica.

Neste estudo, nenhuma criança ou adolescente, avaliada no CAPSi, possuía histórico de internação psiquiátrica . No CAPS AD, apenas um usuário tinha passado por esse tipo de internação. No CAPS Geral, 13,8\% (n=8) dos usuários já tinha sido internado por complicações psiquiátricas.

A Lei $n^{\circ}$. 10.216, de junho de 2001, que dispõe sobre a proteção e os direitos das pessoas portadoras de transtornos psíquicos, prevê a responsabilidade do Estado para desenvolver políticas de saúde mental, assistência e promoção de ações de saúde aos portadores de transtornos mentais, o que sugere a criação de serviços alternativos que possam garantir a atenção ao doente mental, prevenindo sua institucionalização e assegurando seu convívio com a família e a sociedade ${ }^{(11-12)}$.

É notório que, após a implantação dos CAPS, houve redução significativa nas internações por complicações psiquiátricas, culminando na redução dos leitos manicomiais. Contudo, nem sempre é possível evitar tais internações, especialmente quando os recursos extra-hospitalares são exauridos. Dessa forma, deve-se garantir também, nas instituições de internação psiquiátrica, assistência integral, incluindo serviços médicos, de assistência social, psicológica, ocupacional e de lazer ${ }^{(13)}$.

Quanto aos diagnósticos dos usuários dos CAPS, verificou-se que esses variam de acordo com a clientela assistida. A definição do diagnóstico em saúde mental é fundamental para a definição de um plano terapêutico, além disso, é de extrema importância para a epidemiologia na avaliação da prevalência dos transtornos mentais na população. 
Dessa forma, torna-se cada vez mais necessária a ampliação da rede de saúde mental com profissionais capacitados, evitando subnotificações e tratamentos inadequados. A implantação das políticas públicas em saúde mental pode ampliar quantitativa e qualitativamente a clientela assistida, permitindo, assim, o controle de agravos em estágios iniciais.

\section{Conclusões}

Ao se avaliar o perfil epidemiológico da rede de saúde mental em Iguatu, CE, pôde-se constatar que os três Centros de Atenção Psicossocial do município atendem os critérios de funcionamento estabelecidos pelo Ministério da Saúde, assistindo usuários com diversos transtornos mentais e oferecendo atividades de inclusão da família e comunidade durante o tratamento.

Observa-se que a maioria dos prontuários estava preenchida, o que facilitou a coleta de dados. Contudo, torna-se salutar padronizar um modelo de registro que contenha o maior número de informações possíveis dos usuários. Para que isso ocorra, os profissionais devem ser treinados e sensibilizados sobre a importância de manter os registros atualizados e devidamente preenchidos.

Quanto ao encaminhamento dos usuários aos serviços, percebe-se que a maioria foi trazida pelos familiares, confirmando a importância dessa rede social na identificação precoce dos transtornos mentais. A Estratégia Saúde da Família (ESF) e os hospitais gerais também contribuíram significativamente para o encaminhamento de usuários à rede de saúde mental.

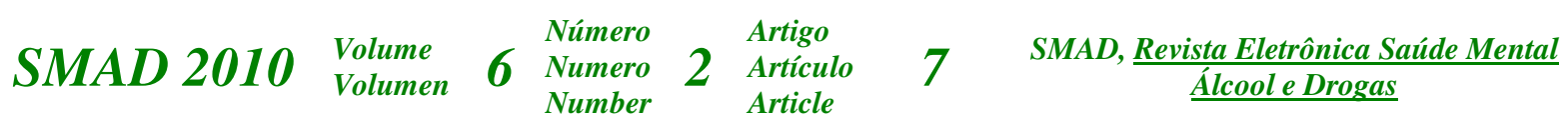


Dessa forma, torna-se relevante fortalecer a dinâmica de referência e contrarreferência entre esses serviços.

A ESF pode contribuir de forma eficaz no acompanhamento de usuários com transtornos leves e moderados, dentro de sua área de abrangência, facilitando a ressocialização desse paciente e mantendo-o próximo de seu convívio familiar.

Em relação às atividades terapêuticas, pode-se constatar que a consulta clínica individual é a mais utilizada nos serviços, sendo seguida pelo desenvolvimento de atividades terapêuticas coletivas, o que aponta avanço na efetivação da clínica ampliada.

A criação e o funcionamento dos CAPS trouxeram nova perspectiva para os usuários dos serviços de saúde mental em Iguatu, CE, aproximando o atendimento e proporcionando assistência especializada, mais humanizada e integral. Nesse contexto, é salutar intensificar ações terapêuticas que envolvam as redes de apoio e que estimulem a inclusão social.

Verifica-se que elevado percentual de usuários faz uso contínuo de psicoterápicos, confirmando essa prática como a principal conduta no atendimento dessa clientela. Dessa forma, torna-se importante efetivar estratégias terapêuticas que promovam a redução do quantitativo de medicamentos utilizados nos tratamentos, sem prejuízos aos usuários.

É notório o esforço da rede de saúde mental do município na tentativa de romper o modelo assistencial ainda vigente, centrado na clínica e na doença. As efetivações de atividades terapêuticas diversificadas nos centros de atenção psicossocial permitem a integração dos usuários em diversas categorias de suporte, possibilitando transformação socioeducacional e humana.

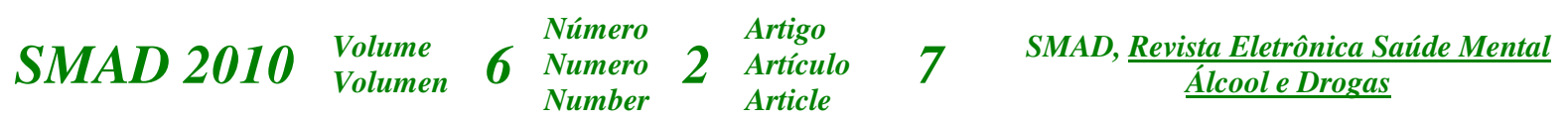


Para que a reforma psiquiátrica ocorra de maneira efetiva, tornam-se fundamentais a valorização dos profissionais da rede e o apoio institucional às atividades planejadas em todos os níveis de gestão.

O município estudado caminha para a assistência em saúde mental de qualidade, valorizando as potencialidades do indivíduo, cooperando efetivamente para a inclusão social e envolvendo diversos setores da sociedade, em busca de melhor qualidade de vida para todos.

\section{Referências}

1. Ministério da Saúde (BR). Guia de saúde mental. Porto Alegre: Ministério da Saúde; 2001.

2. Pontes PAR. Reforma Psiquiátrica no Ceará: descrição de um caso. Rev Latino-Am Enfermagem. 1997; 5(1):45-50.

3. Ministério da Saúde (BR). Secretaria de Atenção à Saúde. Legislação em saúde mental: 1990-2004. 5.ed. Brasília: Ministério da Saúde; 2004.

4. Ministério da Saúde (BR). Secretaria de Atenção à Saúde. Saúde Mental no SUS: acesso ao tratamento e mudança do modelo da atenção. Relatório de Gestão: 2003-2006. Brasília: Ministério da Saúde; 2007.

5. Louzada RCR. Caracterização da clientela atendida no Núcleo de Psicologia Aplicada da Universidade Federal do Espírito Santo. Rev Est Psicol. 2003; 8(3):451-7.

6. IBGE. Instituto Brasileiro de Geografia e Estatística [internet]. Contagem da população 2007. [acesso em: 20 março 2009]. Disponível em: http://www.ibge.gov.br/cidadesat/topwindow.htm?1.

7. Rabasquinho C, Pereira H. Gênero e Saúde mental: uma abordagem epidemiológica. Análise Psicol. 2007; 25(3):439-54.

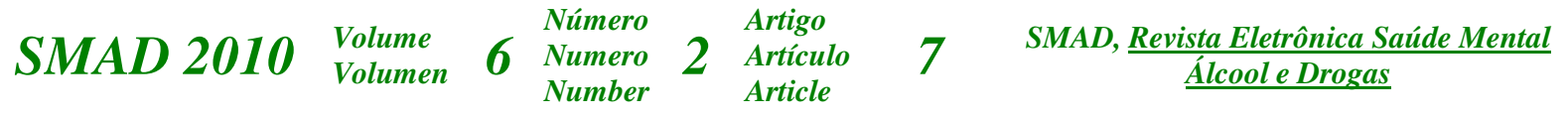


8. Schrank GO. O Centro de Atenção Psicossocial e a inserção da família. [Dissertação Mestrado em Enfermagem]. Porto Alegre (RS): Escola de Enfermagem/UFRS; 2006. 113 p.

9. Ministério da Saúde (BR). Saúde mental no SUS: os Centros de Atenção Psicossocial. Brasília: Ministério da Saúde; 2004. 86 p.

10. Souza AR Centro de Atenção Psicossocial: perfil epidemiológico dos usuários. [Dissertação Mestrado em Enfermagem em Saúde Comunitária]. Faculdade de Odontologia, Farmácia e Enfermagem/UFC; 2007. 97 p.

11. Lei no 10.216, de 6 de abril de 2001 (BR). Dispõe sobre a proteção e os direitos das pessoas portadoras de transtornos mentais e redireciona o modelo assistencial em saúde mental. Brasília; 2001.

12. Britto RC. A internação psiquiátrica involuntária e a Lei 10216-01. Reflexões acerca de garantia de proteção aos direitos da pessoa com transtorno mental. [Dissertação Mestrado em Saúde Pública]. Rio de Janeiro: Escola Nacional de Saúde Pública; 2004. 210 p.

13. Rossato VMD, Melo TAP, Pires FB, Cunha RWP, Silva AA, Freitas FF. Práticas desinstitucionalizadoras em uma unidade de internação psiquiátrica. SMAD. [periódico online]. 2006: 2(2):0-0. [acesso em: 01 maio 2009]. Disponível em: http://pepsic.bvspsi.org.br/pdf/smad/v2n2/v2n2a04.pdf.

Recebido em: 16/02/2010

Aprovado em: 04/05/2010

\section{Como citar este artigo:}

Carvalho MDA, Silva HO, Rodrigues LV. Perfil epidemiológico dos usuários da Rede de Saúde Mental do Município de Iguatu, CE. SMAD, Rev. Eletrônica Saúde Mental Álcool Drog. (Ed. port.) [Internet]. 2010 [acesso em: dia mês abreviado com ponto ano] ; 6(2):337-49. Disponível em: Endereço Eletrônico Visitado.

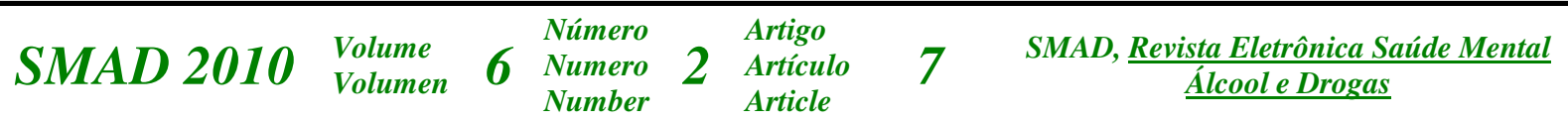

